\title{
Phenotypic variation in progenies from somatic hybrids between Brassica napus and Sinapis alba
}

\author{
Aimin Li · Cunxu Wei · Jinjin Jiang • \\ Yongtai Zhang $\cdot$ Rod J. Snowdon . \\ Youping Wang
}

Received: 29 January 2009/ Accepted: 16 June 2009/Published online: 27 June 2009

(C) The Author(s) 2009. This article is published with open access at Springerlink.com

\begin{abstract}
A number of variant phenotypes, such as long siliques, high density of pods, increased seed number per pod and yellow seed color resembling Sinapis alba, were selected from intergeneric somatic hybrids between Brassica napus and Sinapis alba through successive backcrosses. Resistance to Sclerotinia sclerotiorum among $24 \mathrm{BCF}_{4}$ lines was also tested by inoculation with mycelia, and four lines showed stronger resistance than the resistant rapeseed variety 'Zhongshuang 9'. Comparative anatomy studies on mature seed revealed that the seed coat pigments were mainly distributed in the palisade layer, which is considerably thinner in $S$. alba compared to B. napus. The area index of protein bodies in cotyledon cells was highest in $S$. alba, lowest in B. napus and intermediate in the intergeneric progenies. This study demonstrates that wide hybridization can enable exploitation of valuable trait
\end{abstract}

A. Li · C. Wei · J. Jiang · Y. Wang $(\bowtie)$

College of Bioscience and Biotechnology,

Yangzhou University, 225009 Yangzhou, China

e-mail: wyouping@yahoo.com

A. Li · Y. Zhang

Jiangsu Institute of Agricultural Science in the Lixiahe

District, 225009 Yangzhou, China

R. J. Snowdon

Institute for Plant Breeding and Crop Science I,

Justus Liebig University, Heinrich-Buff-Ring 26-32,

35392 Giessen, Germany diversity from $S$. alba for broadening the genetic basis for rapeseed breeding.

Keywords Brassica napus L. · Intergeneric somatic hybrid · Sclerotinia sclerotiorum .

Sinapis alba L. · Yellow seed

\section{Introduction}

The genetic basis of Brassica napus L., one of the most important oilseed crops worldwide, is quite narrow. The species originated in Europe and was introduced into China as an oil crop in the 1950s (Liu 1984; Wang 2005). Intergeneric hybridization with related crucifer species is often used to broaden genetic variability in B. napus (Snowdon et al. 2006), however, sexual incompatibility limits the use of such approaches (Wang et al. 2005a, b, c). On the other hand, somatic hybridization allows not only the creation of hybrid and cybrid combinations of species that are sexually incompatible, but also recombination and transfer of cytoplasmic genes from both parents, with the aim of improving the characteristics controlled by cytoplasmic genes (Ge and Chen 2004; Liu et al. 2005; Prakash et al. 2009). Protoplast fusion was also used by Ren et al. (2000) and Hu et al. (2002) to recover novel rapeseed germplasm resistant to bacterial soft rot and Leptosphaeria maculans. 
Sinapis alba L., a member of the Brassicaceae, possesses desirable agronomic characteristics such as yellow seed color, tolerance to drought stress, reduced pod shattering (Downey 1987), resistance to virus diseases, blackleg disease, black spot and beet cyst nematodes (Bodnaryk and Lamb 1991; Lelivelt et al. 1993; Brown et al. 1997; Hansen and Earle 1997). Intergeneric hybrids resistant to black spot were produced by ovary culture of $B$. rapa $\times S$. alba sexual hybrids (Gong et al. 1994). Somatic hybrids of $B$. napus and $S$. alba obtained by electrofusion have been described previously (Wang et al. $2005 b, c)$. For creation of high yielding rapeseed lines with improved disease resistance and seed quality, these hybrids were subsequently backcrossed with $B$. napus and self-pollinated to obtain $\mathrm{BCF}_{4}$ generation with valuable agronomic characteristics derived from S. alba. This material contains various interesting phenotypes that may be useful for rapeseed breeding. The objective of this study was to characterize phenotypic variants observed in the $\mathrm{BCF}_{4}$ progenies.

\section{Materials and methods}

\section{Materials}

Intergeneric somatic hybrids (AACCSS, $2 n=62$ ) between $B$. napus and $S$. alba obtained by electrofusion were subsequently backcrossed with $B$. napus cv. 'Yangyou 6' (AACC, $2 n=38$ ) and self-pollinated. Figure 1 shows the backcrossing scheme for

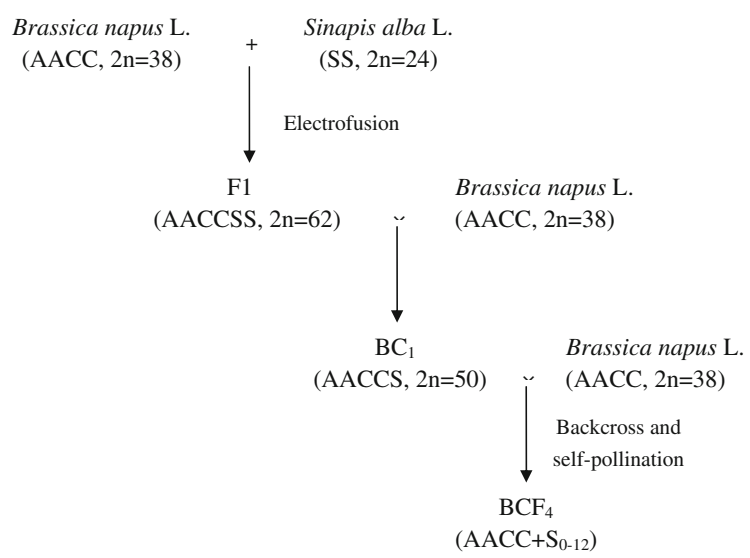

Fig. 1 Backcrossing scheme for the development of $\mathrm{BCF}_{4}$ progenies the development of $\mathrm{BCF}_{4}$ progeny during 2005 and 2008.

Observation of agronomic characteristics

Field tests of the somatic hybrid backcross progeny were carried out at the experimental station of Jiangsu Lixiahe Region Agricultural Research Institute, China. Morphological variations observed in $\mathrm{BCF}_{4}$ included main axis length, number of pods per plant, number of seeds per silique and pod length, as well as the beak length of pods. Pod density was described by the ratio between the number of pods and the length of the main raceme.

Pigment distribution in seed coat and thickness of the palisade layer

Experimental samples were taken from the adaxial side of seeds, then $15 \mu \mathrm{m}$ thick frozen sections were cut using a Leica CM1100 microtome at $-15^{\circ} \mathrm{C}$. Sections were photographed using an Olympus CX 41 light microscope without staining. The thickness of the palisade layer was measured on three seeds from each line, using the parental lines as a control.

\section{Anatomical structure of seeds}

After soaking in $\mathrm{ddH}_{2} \mathrm{O}$ for $1 \mathrm{~h}$ at room temperature, seed capsules from the adaxial side and cotyledon embryos were used as experimental samples for transmission electronic microscopy. Samples were embedded in Spurr's resin, then semi-thin resin sections $(1 \mu \mathrm{m})$ were cut on a Leica EM UC6 microtome using a glass blade, while ultra-thin sections $(70 \mathrm{~nm})$ were generated using a diamond blade. The semi-thin sections were counterstained with PAS-sudan black B-coomassie blue R according to $\mathrm{Hu}$ and $\mathrm{Xu}$ (1990). Observations and photographs were made via light microscopy. The number of protein bodies was counted in ten adjacent cells on each section, and the cumulative area of protein bodies along with the total area of the ten cells was measured using the morphological analysis software JD801. Subsequently, the mean area index of protein body per cell was calculated in each line. All data presented in this paper were obtained from five sections from each of the progeny and parental lines. After double-staining with uranium acetate and lead 
citrate, observation and image merging of the ultrathin sections were done with a Philips Tecnai 12 transmission electronic microscope.

\section{Characterization of Sclerotinia sclerotiorum resistance}

Artificial inoculation of plants with S. sclerotiorum was performed according to Cruickshank et al. (1983). After $72 \mathrm{~h}$ of mycelia inoculation with twenty detached leaves from different plants of each progeny line, the length and width of disease lesions were measured. The level of resistance to $S$. sclerotiorum was judged by the disease lesion area using the formula $S=L \times W$ ( $S$ : lesion area; $L$ : length of lesion; $W$ : width of lesion). All trial data were analyzed using the software DPS.

Determination of oil content

The oil content of self-pollinated seed from each of the $\mathrm{BCF}_{4}$ progeny and parental lines was detected by Near Infrared Reflectance Spectroscopy using the NIRS Model TR-3700 from FOSS (Denmark).

\section{Results}

Variation in pod characteristics

Compared with $\mathrm{BC}_{1}$ plants, pod length and seeds per pod among $\mathrm{BCF}_{4}$ progeny lines were dramatically improved (Table 1). Three $\mathrm{BCF}_{4}$ lines (D214, D224, D249) were identified with distinct variation in pod characteristics. D249 had a very high pod density of $1.8 / \mathrm{cm}, 38.5 \%$ higher than the recurrent parent 'Yangyou 6' (1.3/cm). Lines D214 and D224 had considerably longer siliques than the recurrent $B$. napus parent (Fig. 2e, f). The silique lengths of up to 9.3 and $9.1 \mathrm{~cm}$ in length, respectively, correspond to an increase of 69.1 and $65.5 \%$, respectively compared to 'Yangyou 6' $(5.5 \mathrm{~cm})$. The number of seeds per pod in these three lines was over 26.8, a increase of $46.5,55.2$ and $60.7 \%$ respectively compared to 'Yangyou 6' (18.3 seeds per pod). Furthermore, the pods of the three selected lines had characteristically long beaks (Fig. 2), ranging from 1.4 to $1.8 \mathrm{~cm}$, which resembles the pods of $S$. alba $(2.0 \mathrm{~cm}$ beak length) rather than 'Yangyou 6' (1.1 cm beak length).

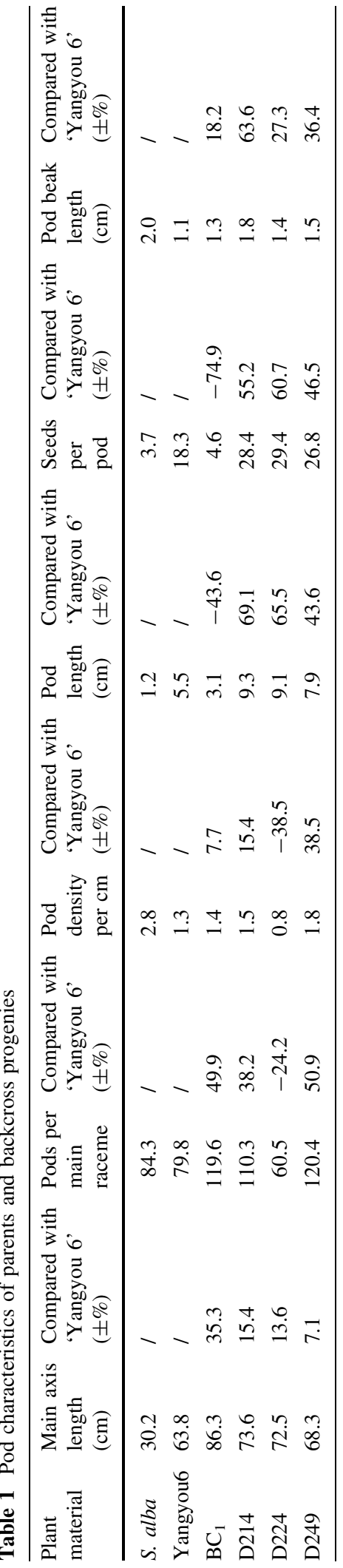


Fig. 2 Comparison of silique among parents, $\mathrm{BC}_{1}$ and $\mathrm{BCF}_{4}$ plants.

a B. napus, b $S$. alba, c $\mathrm{BC}_{1}$, d-f $\mathrm{BCF}_{4}$ lines, d D249, e D214, f D224
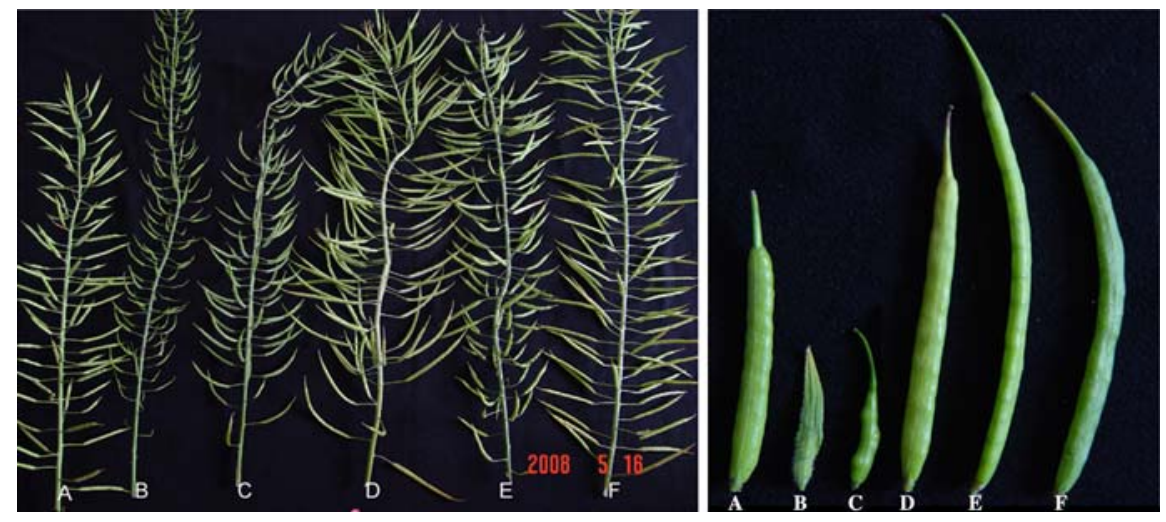

Fig. 3 Seed coat color of backcross progenies and their parents. a $S$. alba, b 'Yangyou 6', c D244-18, d D244-52, e D244-6, f D255-3, $\mathbf{g}$ D246-5, h D211
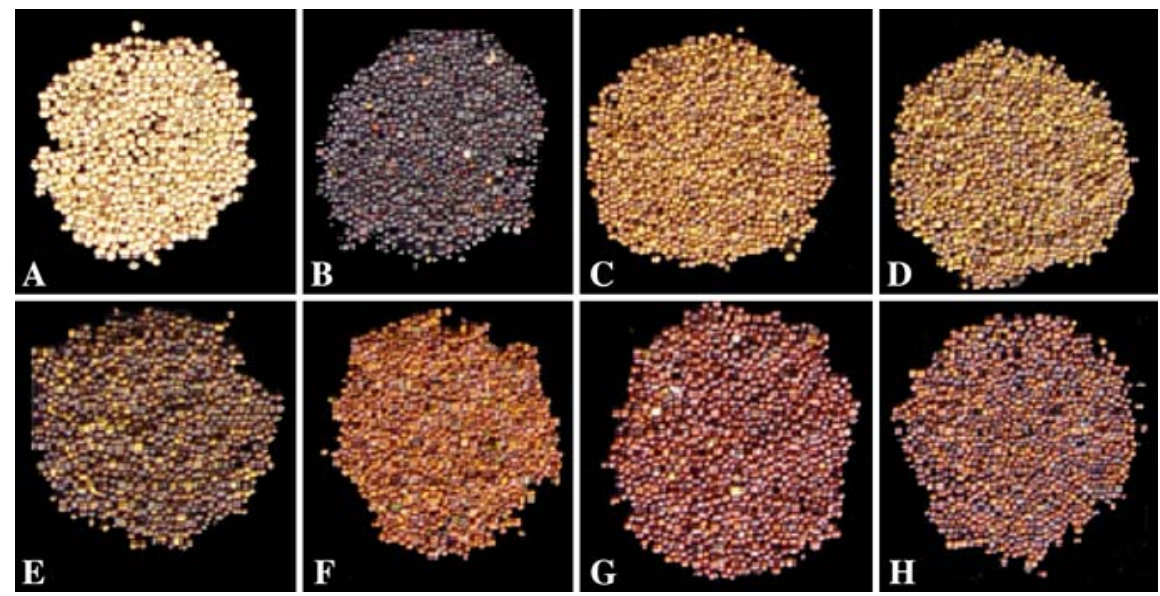

Oil content and anatomical structure of yellow seeded progenies

Several lines with yellow seed color derived from the S. alba parent were identified among the backcross progenies. The seed color of these lines ranged from brownish yellow to yellow, while the $B$. napus parents had dark brown seeds (Fig. 3a-h). The oil content of these six yellow-seeded lines was found to be increased to $43.3,43.4,47.6,42.9,42.8,44.6 \%$, respectively, compared to the oil content of $41.6 \%$ in 'Yangyou6' and $39.0 \%$ in the S. alba parental line.

The seed coat pigments were mainly distributed in the palisade layer (Fig. 4), the highest quantity of seed coat pigmentation was observed in 'Yangyou6', followed by the backcross lines D246-5, D211, D255-3 and D244-52. On the other hand, the seeds of the $S$. alba parent and the backcross progenies D244-18 and D244-6 exhibited almost no pigmentation. The thickness of palisade layer was highest in
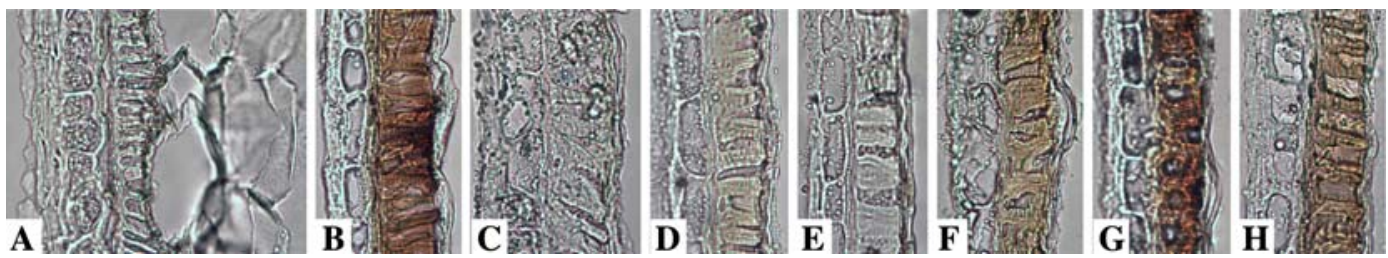

Fig. 4 Pigment distribution in seed coat of backcross progenies and their parents. a S. alba, b 'Yangyou 6', c D244-18, d D244-52, e D244-6, f D255-3, g D246-5, h D211. ×200 


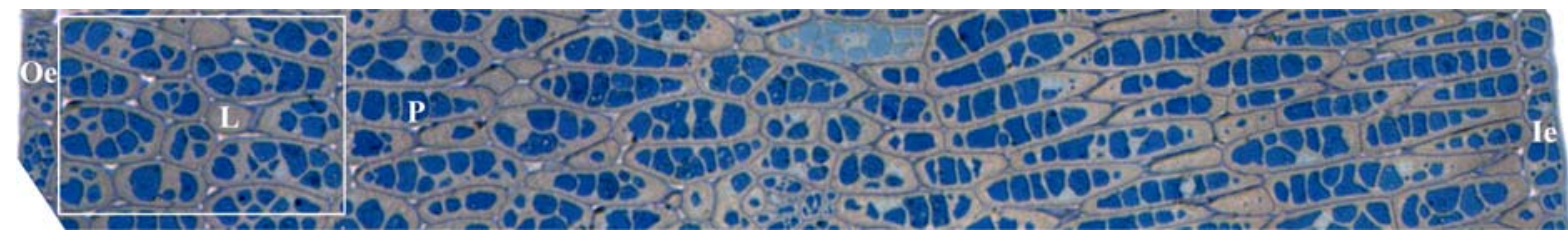

Fig. 5 The semi-thin section of cotyledon in $S$. alba. $\times 250$. Ie inner epidermical cell, $L$ lipid body, $O e$ outer epidermical cell, $P$ protein body

B. napus, thinnest in S. alba and intermediate in the hybrid progenies (Fig. 4).

When treated by PAS-sudan black B-coomassie blue $\mathrm{R}$, the lipid body and storage proteins on semithin sections of cotyledon embryos were dyed gray and blue, respectively. The first step of PAS staining stains cell walls red, however, this changes to blueblack after sudan black B and coomassie blue $\mathrm{R}$ counterstaining (Fig. 5). Statistical analysis of the protein body area index in the first three layers under the outer epidermical of the seed cotyledons (Fig. 5) revealed that the protein body area index in cotyledon cells was highest in $S$. alba, lowest in B. napus and intermediate in progenies (Fig. 6).

\section{Characterization of resistance to Sclerotinia sclerotiorum}

Statistical analysis of disease lesions in the twentyfour progeny lines tested showed a significant variation in disease response among lines after inoculation with mycelia $(F=16.693, v 1=494$, $v 2=25$ ). Compared with line D267 and 'Zhongshuang 9', which were used as susceptible and resistant controls, respectively, different resistance reactions were observed among the tested lines. Through multicomparison of inoculation of detached leaves with mycelia, we founded no significant difference in lines

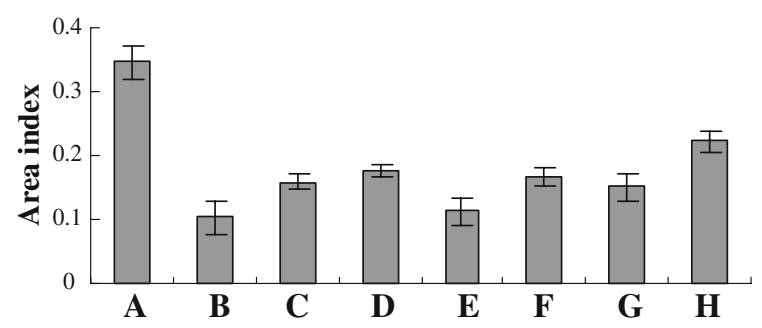

Fig. 6 Area index of protein body of backcross progenies and their parents a $S$. alba, b 'Yangyou 6', c D244-18, d D244-52, e D244-6, f D255-3, g D246-5, h D211
D216, D222, D225, D226 and D220 compared with susceptible line D267. On the other hand, lines D214, D245 and D219 were shown to have a stronger resistance than D267, but weaker than 'Zhongshuang 9'. Another eleven lines (D218, D223, D246, D212, D265, D211, D273, D241, D230, D237 and D213) were confirmed to have no obvious difference from the resistant control. Lines D244, D243, D229 and D240 exhibited the strongest resistance to S. sclerotiorum and showed significant differences to the resistant control 'Zhongshuang 9' (Table 2).

\section{Discussion}

Distant hybridization of sexually incompatible plant species via somatic fusion can overcome intergeneric boundaries, enlarge genetic variation, and ultimately result in the creation of new variants and species that do not occur naturally. Both sexual and somatic hybridization have been widely used to introduce traits of interest from related crucifer species to oilseed rape (Snowdon et al. 2006). Roy (1984) and Lu et al. (2006) obtained novel rapeseed with yellow seed color and resistance to blackleg through hybridization between $B$. juncea and B. napus, respectively. Progenies with extreme pod length variation $(20 \mathrm{~cm}$ in length) were also created from interspecific hybrids of B. rapa and B. oleracea (Niu et al. 2005). These examples demonstrate that distant hybridization can not only introduce new characteristics from donors into phylogenetically close plants, but also create new variant types beyond the variation present in the parental species. In this study, we obtained lines with many agronomically interesting phenotypes from intergeneric somatic hybrid progenies of $B$. napus and $S$. alba through successive backcrosses. These included lines wth high pod density, yellow seed coats, resistance to Sclerotinia sclerotiorum, long siliques, increased numbers of seeds per pod and 
Table 2 Multi-comparison of disease lesion areas after artificial inoculation of detached leaves with Sclerotina sclerotiorum mycelia (PLSD)

\begin{tabular}{llll}
\hline Line No. & Mean lesion $\left(\mathrm{cm}^{2}\right)$ & Significance at $1 \%$ & Significance at $1 \%$ \\
\hline D216 & 25.12 & a & A \\
D222 & 24.98 & a & A \\
D267 & 24.65 & ab & AB \\
D225 & 23.16 & abc & ABC \\
D226 & 21.66 & bcd & ABC \\
D220 & 21.39 & bcd & ABC \\
D214 & 20.90 & cd & ABCD \\
D245 & 20.29 & cd & BCDE \\
D219 & 20.27 & cd & BCDE \\
D215 & 18.74 & de & CDEF \\
D218 & 16.69 & ef & DEFG \\
D223 & 16.53 & ef & DEFG \\
D246 & 16.22 & efg & EFG \\
D212 & 15.51 & efgh & FG \\
Zhongshuang9 & 15.15 & fgh & FG \\
D265 & 14.53 & fgh & FGH \\
D211 & 14.44 & fgh & FGH \\
D273 & 14.23 & fgh & FGH \\
D241 & 13.26 & fghi & GH \\
D230 & 12.85 & ghi & GH \\
D237 & 12.74 & ghi & GHI \\
D213 & 12.08 & hi & GHI \\
D244 & 10.17 & ij & HIJ \\
D243 & 9.88 & j & HIJ \\
D229 & 8.07 & & IJ \\
D240 & 6.85 & J \\
\hline & & & \\
\hline
\end{tabular}

improved oil content compared to the B. napus parent. These variant progenies are of great interest for rapeseed breeding, offering abundant germplasm resources for the creation of new varieties with improved disease resistance, seed quality and yield.

The oil and protein content of the yellow seeds is often higher than that of black ones to the the increased contribution of the cotyledons to the overall seed volume. In addition, the thinner seed coat of yellow-seeded B. napus is also associated with a lower antinutritive fiber content, while the reduced pigmentation results in a clear and transparent oil and a higher palatability of the seed meal in animal feeds. The yellow-seeded progenies obtained in the present study were found to have a thinner seed coat and reduced pigmentation compared to the dark-seeded B. napus parent. In contrast, the oil bodies of the best progenies resembled oil bodies in B. napus seeds rather than the smaller oil bodies of $S$. alba which has a very low oil content. On the other hand, the protein body area was significantly reduced in seeds from yellow-seeded progenies with high oil content, indicating that the reduction in seed coat thickness resulted in an increase in oil rather than an increase in protein proportion in the seeds.

Until now, genetic variation for seed color was only introduced to $B$. napus by interspecific hybridization with the closely related Brassica species $B$. rapa, B. carinata, B. juncea and $B$. oleracea. The seed color of $S$. alba is however, considerably lighter than in most Brassica species, raising the possibility that the yellow-seed trait derived from $S$. alba could further improve the meal and oil quality traits associated with light-seeded rapeseed. The progenies identified in the present study combine the yellow seed trait from $S$. alba with other agronomic 
properties that could improve the oil content, seed yield and seed quality in B. napus, and are therefore of considerable interest for rapeseed breeding.

Sinapis alba is phylogenetically close to Brassica A and C genomes (Warwick and Black 1991), and has sufficient genetic homoeology (Gaikward et al. 1996). Nelson et al. (2005) found that $95 \%$ of the Brassica DNA fragments could be hybridized strongly to low copy-number sequences in the $S$. alba genome. Formation of multivalents and chromosome recombination in the progenies derived from B. napus and S. alba hybrid (Wang et al. 2005c) is also indicative of intergenomic homoeology between both $S$. alba and A/C genomes. The phenotypic variation presented herein with different agronomical characteristics from $\mathrm{BCF}_{4}$ progenies to $\mathrm{BCF}_{5}$ exhibited almost stable. One reason is these progenies had a very good fertility and a high seedset. The other reason is these progenies had 38 chromosomes and exhibited a normal meiosis (data not shown). How to identify these introgression lines, especially with yellow seeds, using molecular markers and to use this valuable germplasm for rapeseed breeding is our present task.

Acknowledgments This research was supported by the National Key Project 973 (2006CB101603) and the National Natural Science Foundation of China (30671166).

Open Access This article is distributed under the terms of the Creative Commons Attribution Noncommercial License which permits any noncommercial use, distribution, and reproduction in any medium, provided the original author(s) and source are credited.

\section{References}

Bodnaryk RP, Lamb RJ (1991) Mechanisms of resistance to the flea beetle, Phyllotreta cruciferae (Goeze), in yellow mustard seedlings, Sinapis alba L. Can J Plant Sci 71:1320

Brown J, Brown AP, Davis JB, Erickson D (1997) Intergeneric hybridization between Sinapis alba and Brassica napus. Euphytica 93:163-168

Cruickshank RH (1983) Distinction between Sclerotinia species by their pectic zymograms. Trans Br Mycol Soc 80:117-119

Downey RK (1987) Rapeseed and mustard. In: Fehr WR (ed) Principles of cultivar development. Macmillan Publishing Company, New York, pp 437-486
Gaikward K, Kirti PB, Sharma A, Prakash S, Chopra VL (1996) Cytogenetical and molecular investigations on somatic hybrids of Sinapis alba and Brassica juncea and their backcross progeny. Plant Breed 115:480-483

Ge YM, Chen LP (2004) Progress of plant cell engineering germplasm enhancement of Cruciferae. Chin J Cell Biol 26:471-474

Gong ZH, He YK, Wang M (1994) Studies on the resistance of intergeneric hybrids cabbage $\times$ white mustard to Alternaria leaf spot. Acta Hort Sin 21:401-403

Hansen LN, Earle ED (1997) Somatic hybrids between Brassica oleracea L. and Sinapis alba L. with resistance to Alternaria brassicae (Berk.) Sacc. Theor Appl Genet 94:1078-1085

Hu SY, Xu LY (1990) A cytochemical technique for demonstration of lipids, polysaccharides and protein bodies in thick resin sections. Acta Bot Sin 32:841-846

Hu Q, Anderson SB, Dixelius C, Hansen LN (2002) Production of fertile intergeneric somatic hybrids between Brassica napus and Sinapis arvensis for the enrichment of the rapeseed gene pool. Plant Cell Rep 21:147-152

Lelivelt CLC, Leunissen EHM, Frederiks HJ, Helsper JPFG, Krens FA (1993) Transfer of resistance to the beet cyst nematode (Heterodera schachtii $\mathrm{Schm}$.) from Sinapis alba $\mathrm{L}$. (white mustard) to the Brassica napus L. gene pool by sexual and somatic hybridization. Theor Appl Genet 85:688-696

Liu HL (1984) Origin and evolution of some Brassicas. Acta Agron Sin 109:9-18

Liu J, Xu X, Deng X (2005) Intergeneric somatic hybridization and its application to crop genetic improvement. Plant Cell Tiss Org Cult 82:19-44

Lu KC, Liu SY, Guo JB (2006) Development of the novel yellow-seeded Brassica napus germplasm through the interspecific crosses B. juncea $\times$ B. napus. J Hunan Agric Univ 32:116-119

Nelson MN, Nixon J, Lydiate DJ (2005) Genome-wide analysis of the frequency and distribution of crossovers at male and female meiosis in Sinapis alba L. (white mustard). Theor Appl Genet 111:31-34

Niu YZ, Guo SX, Fu SH (2005) Development of a speciallylong pod variant line from resynthesized Brassica napus L. J Plant Genet Res 6:151-155

Prakash S, Bhat SR, Quiros CF, Kirti PB, Chopra VL (2009) Brassica and its close allies: cytogenetics and evolution. Plant Breed Rev 31:21-187

Ren JP, Dickson MH, Earle ED (2000) Improved resistance to bacterial soft rot by protoplast fusion between Brassica rapa and B. oleracea. Theor Appl Genet 100:810-819

Roy NN (1984) Interspecific transfer of Brassica juncea-type high blackleg resistance to Brassica napus. Euphytica 33:295-303

Snowdon RJ, Lühs W, Friedt W (2006) Oilseed rape. In: Kole $\mathrm{C}$ (ed) Genome mapping and molecular breeding, vol 2: oilseeds. Springer Verlag, Heidelberg, pp 55-114

Wang HZ (2005) Problem in the development of oilseed industry and it's countermeasure in China. Chin J Oil Crop Sci 27:100-105

Wang AY, Li X, Hu DY (2005a) Research advances on distant hybridization breeding in rapeseed. Acta Agriculturae Boreali-occidentalis Sin 14(6):67-71 
Wang YP, Sonntag K, Rudloff E, Chen JM (2005b) Intergeneric somatic hybridization between Brassica napus and Sinapis alba. J Integr Plant Biol 47:84-91

Wang YP, Zhao XX, Sonntag K, Wehling P, Snowdon RJ (2005c) Behaviour of Sinapis alba addition chromosomes in a Brassica napus background revealed by genomic in situ hybridisation. Chromosom Res 13:819-826
Warwick SI, Black LD (1991) Molecular systematics of Brassica and allied genera (Subtribe Brassinae, Brassiceae) chloroplast genome and cytodeme congruence. Theor Appl Genet 82:81-92 